\title{
WILANSKY'S QUERY ON OUTER MEASURES
}

\section{ChOO-Whan KIM}

\begin{abstract}
On a set $X$, let $\mu^{*}$ be an outer measure and $\mu$ the measure induced by $\mu^{*}$. We show that if $X$ is a finite set, then the measure $\mu$ is saturated. We give two examples of non-regular outer measures on an infinite set $X$ which induce non-saturated and saturated measures, respectively. These answer a query posed by Wilansky.
\end{abstract}

\section{Introduction and preliminaries}

Let $X$ be an arbitrary non-empty set and $P(X)$ its power set. On the set $X$, let $\mu^{*}$ be an outer measure, $\mu$ the measure induced by $\mu^{*}$, $\mu^{+}$the outer measure induced by $\mu$, and $\bar{\mu}$ the measure induced by $\mu^{+}$. Recently Wilansky posed the following query [3]: must every $\mu^{+}$-measurable set be $\mu^{*}$-measurable?

Let $M$ and $M^{+}$be the $\sigma$-algebras of $\mu^{*}$-measurable and $\mu^{+}$-measurable sets, respectively. It is plain that $M \subset M^{+}, \mu^{*} \leq \mu^{+}$on $P(X)$, and $\mu^{*}=\mu^{+}$on $M$.

Let $(X, B, \lambda)$ be any measure space. Following Royden [2] we shall say that a subset $E$ of $X$ is locally measurable (with respect to $B$ and $\lambda$ ), if $E \cap B \in B$ for each $B \in B$ with $\lambda(B)<\infty$. Then the family $B^{\wedge}$ of all locally measurable sets is a $\alpha$-algebra containing $B$. The measure $\lambda$ is called saturated (or a saturated measure on $B$ ), if $B=B^{\wedge}$. If $\lambda$

Received 24 October 1984.

Copyright Clearance Centre, Inc. Serial-fee code: 0004-9727/85 $\$$ A2. $00+0.00$. 
is a $\sigma$-finite measure, then it is saturated. For any subset $E$ of $X$, let $E^{\prime}=X-E$.

LEMMA 1. If $E \in M^{+}$with $\bar{\mu}(E)<\infty$, then $E \in M$.

Proof. Suppose $E \in M^{+}$with $\bar{\mu}(E)<\infty$. Let $A \in M$ be such that $E \subset A$ and $\bar{\mu}(E)=\mu(A)$. Then $A-E \in M^{+}$and $\bar{\mu}(A-E)=0$, so that $\mu(A-E)=0$. Since $\mu$ is complete, we have $A-E \in M$, so that $E \in M . \square$

LEMMA 2. $M^{\wedge}=\left(M^{+}\right)^{\wedge}$.

Proof. Suppose $E \in\left(M^{+}\right)^{\wedge}$ and $B \in M$ with $\mu(B)<\infty$. Then we have $E \cap B \in M^{+}$and $\bar{\mu}(E \cap B) \leq \mu(B)<\infty$, so, by Lemma $I, E \cap B \in M$. Thus $\left(M^{+}\right)^{\wedge} \subset M^{\wedge}$. Similarly we obtain $M^{\wedge} \subset\left(M^{+}\right)^{\wedge}$.

PROPOSITION 1. $\bar{\mu}$ is a saturated measure on $M^{+}$, and $M^{+}=M^{\wedge}$.

Proof. Since $M^{+} \subset\left(M^{+}\right)^{\wedge}=M^{\wedge}$, it remains to show $M^{\wedge} \subset M^{+}$. Suppose $E \in M^{\wedge}$ and $A \subset X$ with $\mu^{+}(A)<\infty$. Let $B \in M$ be such that $A \subset B$ and $\mu^{+}(A)=\mu(B)$. Then both $E \cap B$ and $E^{\prime} \cap B$ are in $M$, and $\mu^{+}(A)=\mu(B)=\mu(B \cap E)+\mu\left(B \cap E^{\prime}\right) \geq \mu^{+}(A \cap E)+\mu^{+}\left(A \cap E^{\prime}\right)$, so that $E \in M^{+}$.

In view of Proposition 1, the query may be stated as follows: must the measure $\mu$ induced by an outer measure $\mu^{*}$ be saturated?

\section{Results}

We state without proof the following well-known result ([1], [2]).

PROPOSITION 2. The following assertions are equivalent:

(i) $\mu^{*}=\mu^{+}$on $P(X)$;

(ii) for each $E \subset X$, there is $A \in M$ such that $E \subset A$ and $\mu^{*}(E)=\mu^{*}(A)$;

(iii) $\mu^{*}$ is induced by a measure on an algebra.

An outer measure $\mu^{*}$ is called regular, if any one of the assertions of Proposition 2 holds. By a minor modification of the proof of Proposition 1 we obtain:

THEOREM 1. If an outer measure $\mu^{*}$ is regular, then the induced measure $\mu$ is saturated. 
THEOREM 2. For each outer measure $\mu^{*}$ on a finite set $X$, the induced measure $\mu$ is saturated.

Proof. It is enough to prove the theorem in the case in which $\mu^{*}$ is not regular, $\mu^{*}(X)=\infty$, and $X$ has at least three points. Let $X=\{1,2, \ldots, n\} \quad(n \geq 3), Y=\left\{i \mid i \in X, \mu^{*}(i)<\infty\right\}$, and $Z=\left\{i \mid i \in X, \mu^{*}(i)=\infty\right\}$. By our assumption, $Y$ must contain at least two points and $Z$ is not empty. It follows at once that every subset of $Z$ is $\mu^{*}$-measurable. Since $Y$ also is $\mu^{*}$-measurable, we have

$$
M=\{E \cup F \mid E \in Y \cap M, F \subset Z\} \text {. }
$$

If $A \in M^{\wedge}=M^{+}$, then $A \cap Z \in M$ and $A \cap Y \in M^{+}$. Since $\bar{\mu}(A \cap Y) \leq \mu(Y)<\infty$, it follows from Lemma $l$ that $A \cap Y \in M$. Thus $A \in M$.

\section{Examples}

Here we give two examples of non-regular outer measures on an infinite set which induce non-saturated and saturated measures, respectively.

EXAMPLE 1. Let $X$ be an infinite set. Define the outer measure $\mu^{*}$ by

$$
\begin{aligned}
& \mu^{*}(A)=1-2^{-n}, \text { if } A \text { contains } n \text { points, } \\
& \mu^{*}(A)=\infty \text {, if } A \text { is infinite. }
\end{aligned}
$$

For each non-empty proper subset $E$ of $X$, let $A=\{x, y\}$, where $x \in E$ and $y \in E^{\prime}$. Since

$$
\mu^{*}(A \cap E)+\mu^{*}\left(A \cap E^{\prime}\right)=\mu^{*}(x)+\mu^{*}(y)=1>\mu^{*}(A)=3 / 4,
$$

the set $E$ is not in $M$. Thus $M=\{\varnothing, X\}$.

It follows readily that $\mu^{+}(\varnothing)=0$, and $\mu^{+}(E)=\mu(X)=\infty$ for each $E \neq \varnothing$. That is, $\mu^{+}$is the "infinite" measure on $P(X)$. It is plain that $M^{+}=P(X)$ so that the induced measure $\mu$ is not saturated.

EXAMPLE 2. Let $X$ be an infinite set, $Y=\{a, b\}$, where $a$ and $b$ are distinct points of $X$, and $Z=X-Y$. Define the outer measure $\mu^{*}$ by

$$
\mu^{*}(\emptyset)=0, \mu^{*}(a)=\mu^{*}(b)=1, \mu^{*}(Y)=1.5, \mu^{*}(A)=\infty,
$$


Note that both $\{a\}$ and $\{b\}$ are not in $M$. It is straightforward to show that $Y \in M$, and $E \in M$ for all $E \subset Z$. Thus we obtain

$$
M=\{\varnothing, Y, E, Y \cup E \mid \emptyset \neq E \subset Z\} .
$$

Since $\mu^{+}(a)=\mu^{+}(b)=\mu(y)=1.5$, we have

$$
\mu^{+}(Y \cap\{a\})+\mu^{+}(Y \cap\{b\})=2 \mu(Y)>\mu(Y),
$$

so that both $\{a\}$ and $\{b\}$ are not in $M^{+}$. Thus $M=M^{+}$, and the measure $\mu$ is saturated.

\section{References}

[1] Paul R. Halmos, Measure theory (Van Nostrand, New York, 1954).

[2] H.L. Royden, Real analysis, 2nd ed. (Macmillan, New York, 1968).

[3] A. Wilansky, "Query 305", Notices Amer. Math. Soc. 31 (1984), 376.

Department of Mathematics and Statistics,

Simon Fraser University,

Burnaby,

British Columbia V5A IS6,

Canada. 